\title{
Burnout em trabalhadores de enfermagem de hospitais: estudo de tendências
}

\author{
Burnout in hospital nursing workers: trend study \\ Burnout em los trabajadores de enfermería del hospital: estudio de tendencias
}

\author{
Ademir Jones Antunes Dorneles \\ ORCID: https://orcid.org/0000-0002-8251-4202 \\ Universidade Federal de Santa Maria, Brasil \\ E-mail: majademir10@gmail.com \\ Graziele de Lima Dalmolin \\ ORCID: https://orcid.org/0000-0003-0985-5788 \\ Universidade Federal de Santa Maria, Brasil \\ E-mail: grazi.dalmolin@gmail.com \\ Mariane Buligon Facin \\ ORCID: https://orcid.org/0000- 0002-2284-7232 \\ Universidade Federal de Santa Maria, Brasil \\ E-mail: marianebuligon@gmail.com
}

\begin{abstract}
Resumo
Objetivo: conhecer a tendência da produção científica brasileira sobre burnout em trabalhadores de enfermagem no ambiente hospitalar. Método: Revisão narrativa com busca sistemática de teses e dissertações; realizada no período entre setembro de 2020 e março de 2021, a partir do Catálogo de Teses e Dissertações da Coordenação de Aperfeiçoamento de Pessoal de Nível Superior (CAPES), com a combinação das palavras-chaves: "burnout AND "hospital" AND enfermagem". Resultados: Foram incluídos 37 estudos, entre os anos de 2005 e 2019; evidenciaramse três categorias temáticas: burnout e organização do trabalho, fatores associados ao burnout e estratégias e ações para prevenir o burnout. Observou-se que existem fatores correlacionados ente o burnout, a enfermagem e o ambiente hospitalar destacando-se entre eles, a baixa realização no trabalho, a exaustão profissional e o clima ético negativo; que investir. Conclusão/Considerações Finais: investir na prática de enfermagem em ambientes de trabalho éticos e positivos contribuirá na melhoria da qualidade da assistência de enfermagem e na redução de doenças laborais, como o burnout.
\end{abstract}

Palavras-chave: Burnout psicológico; Enfermagem; Hospitais; Saúde do trabalhador.

\begin{abstract}
Objective: to know the trend of Brazilian scientific production on burnout in nursing workers in the hospital environment. Method: Narrative review with systematic search for theses and dissertations; carried out between September 2020 and March 2021, from the Theses and Dissertations Catalog of the Coordination for the Improvement of Higher Education Personnel (CAPES), with the combination of the keywords: "burnout AND "hospital" AND nursing". Results: 37 studies were included, between the years 2005 and 2019; Three thematic categories were highlighted: burnout and work organization, factors associated with burnout and strategies and actions to prevent burnout. It was observed that there are factors correlated between burnout, nursing and the hospital environment, highlighting among them, low performance at work, professional exhaustion and negative ethical climate; to invest. Conclusion/Final Considerations: investing in nursing practice in ethical and positive work environments will contribute to improving the quality of nursing care and reducing work-related illnesses such as burnout.
\end{abstract}

Keywords: Psychological burnout; Nursing; Hospitals; Worker's health.

\begin{abstract}
Resumen
Objetivo: conocer la tendencia de la producción científica brasileña sobre el burnout en trabajadores de enfermería en el ámbito hospitalario. Método: Revisión narrativa con búsqueda sistemática de tesis y disertaciones; realizado entre septiembre de 2020 y marzo de 2021, del Catálogo de Tesis y Disertaciones de la Coordinación para la Perfeccionamiento del Personal de Educación Superior (CAPES), con la combinación de las palabras clave: "burnout Y" hospital "Y enfermería". Resultados: Se incluyeron 37 estudios, entre los años 2005 y 2019; Se destacaron tres categorías temáticas: burnout y organización del trabajo, factores asociados al burnout y estrategias y acciones para prevenir el burnout. Se observó que existen factores correlacionados entre el burnout, la enfermería y el ambiente hospitalario, destacando entre ellos, bajo desempeño en el trabajo, agotamiento profesional y clima ético negativo; para invertir. Conclusión / Consideraciones finales: invertir en la práctica de la enfermería en entornos laborales éticos y positivos contribuirá a mejorar la calidad de la atención de enfermería y reducir las enfermedades relacionadas con el trabajo como el agotamiento.
\end{abstract}

Palabras clave: Burnout psicológico; Enfermería; Hospitales; Salud del trabajador. 


\section{Introdução}

Atualmente, quando se fala sobre doenças laborais, logo vem à palavra "burnout" na discussão; essa associação ocorre como consequência da elevada prevalência dessa síndrome entre os trabalhadores; mostrando a associação entre a prática do trabalho e o burnout (Porciuncula, Venâncio \& Silva, 2020; Alvares, et al., 2020).

Conceitua-se trabalho como uma atividade em que o homem usa sua força para produzir os meios de sustento e propósitos de vida. Entretanto, assim como origina satisfação e realização também causa desgastes ao trabalhador, como o burnout (Porciuncula, et al., 2020; Dubale, et al.,2019).

Define-se burnout como algo que parou de funcionar por exaustão de energia no trabalhador em decorrência da resposta a estressores crônicos da prática do trabalho; descrevendo-se por sinais de exaustão emocional, despersonalização e baixa realização no trabalho (Porciuncula, et al., 2020; Dubale, et al., 2019; Abu Zied, et al., 2020).

Evidencia-se a ocorrência de burnout em trabalhadores de diversas áreas; mas principalmente nas profissões com atividades próximas aos clientes; como professores, bancários e trabalhadores da saúde e enfermagem, entre outros (Porciuncula, et al., 2020; Abu Zied, et al.,2020; Dorneles, Dalmolin, Andolhe, Magnago \& Lunardi, 2020).

$\mathrm{Na}$ enfermagem, a vulnerabilidade ao burnout advém da sua prática cotidiana estar muito perto dos pacientes e familiares no ambiente hospitalar; o que pode levar a alto nível de estresse nesses profissionais (Porciuncula, et al., 2020; Dorneles, et al., 2020). Relacionados aos aspectos do próprio processo de trabalho nesse cenário, o que necessita ainda de aprofundamentos, pois existem aspectos que precisam ser avaliados.

Assim, diante de toda essa problemática, buscou-se responder a seguinte questão de pesquisa: Qual a tendência da produção científica brasileira sobre burnout e enfermagem no ambiente hospitalar, nas dissertações e teses?

Nesse sentido, o presente artigo tem por objetivo conhecer a tendência da produção científica brasileira sobre burnout e enfermagem no ambiente hospitalar nas dissertações e teses.

\section{Metodologia}

Trata-se de uma revisão narrativa com busca sistemática de teses e dissertações, realizada no período entre setembro de 2020 e março de 2021, a partir do Catálogo de Teses e Dissertações da Coordenação de Aperfeiçoamento de Pessoal de Nível Superior (CAPES) (Martins \& Tourinho, 2017; Gonçalves \& Corrêa, 2020).

Acentua-se que uma revisão narrativa não emprega critérios explícitos e sistemáticos para a investigação e análise crítica da literatura; a procura pelas pesquisas não precisa esgotar as fontes de informações; a escolha dos estudos e a interpretação das informações podem estar sujeitas à subjetividade dos autores (Martins \& Tourinho, 2017; Gonçalves \& Corrêa, 2020).

O levantamento bibliográfico de teses e dissertações envolveu atividades de busca, identificação, fichamento e análise dos estudos. Foram analisados resumos de teses e dissertações, sendo utilizadas palavras-chaves com as seguintes estratégias de busca: "burnout AND "hospital" AND enfermagem".

A partir das palavras-chaves, identificaram-se os títulos e resumos de todas as teses e dissertações, selecionando-se aquelas que se enquadravam aos seguintes critérios de inclusão: resumos de teses e dissertações que abordassem sobre burnout e enfermagem no ambiente hospitalar, conforme demonstrado na Figura 1.

Os estudos foram analisados na íntegra e para posterior extração dos dados se utilizou a análise de conteúdo temática; que consiste nas fases de pré-análise, exploração do material, tratamento e interpretação dos resultados (Martins \& Tourinho, 2017; Gonçalves \& Corrêa, 2020). Não houve limitação do período de publicação dos estudos pesquisados. 
Figura 1. Fluxograma de representação da busca realizada no catálogo da CAPES e estudos selecionados.

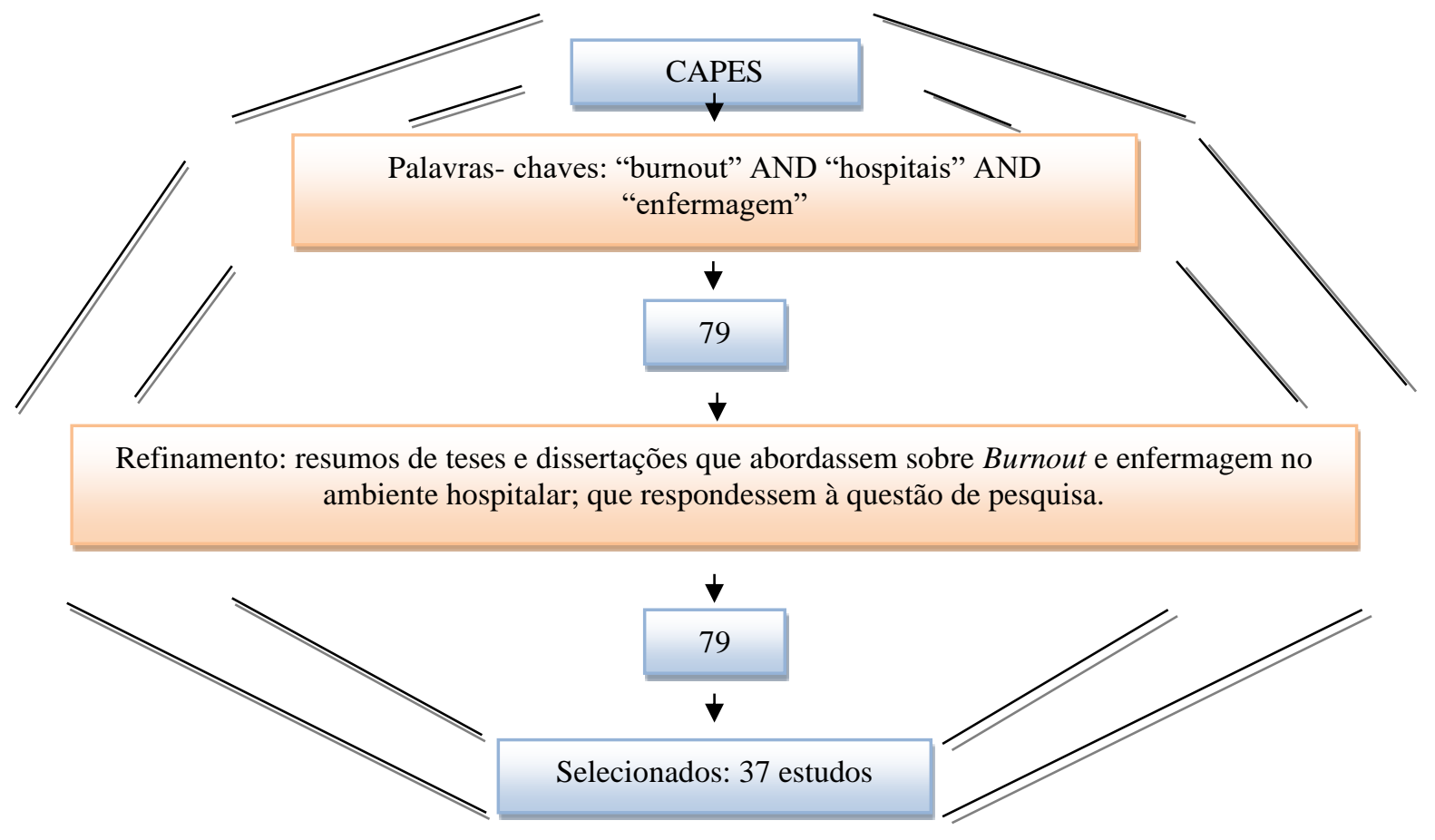

Fonte: Elaboração própria.

\section{Resultados}

Como resultados da busca foram encontrados 79 estudos no Catálogo da CAPES. Na primeira etapa foram selecionados, por dupla escolha independente, os resumos das teses e dissertações que abordassem a temática. Na segunda etapa, foram escolhidos os estudos para análise.

Foram eleitos e analisados 37 estudos relacionados com a temática (cinco teses e 32 dissertações); desses, um era qualitativo (dissertação), quatro eram quali-quantitativos (quatro dissertações) e 32 eram quantitativos (cinco teses e 27 dissertações).

Em relação ao ano de publicação, um era de 2005 (dissertação), um de 2008 (dissertação), um de 2010 (dissertação), cinco de 2011 (dissertações), um de 2012 (dissertação), quatro de 2013 (dissertações), três de 2014 (dissertações), oito de 2015 (três teses e cinco dissertações), dois de 2016 (dissertações) seis de 2017 (duas teses e quatro dissertações) e cinco de 2019 (dissertações).

Com referência a região de procedência desses estudos, um era da região norte (dissertação), dois da região centrooeste (dissertação), quatro da região sul (dissertações), sete da região nordeste (dissertações) e 23 da região sudeste (cinco teses e 18 dissertações).

No Quadro 1 são apresentados os 37 estudos relacionados com burnout, enfermagem e hospital, encontrando-se datado em 2005 o primeiro trabalho identificado (dissertação). 
Quadro 1. Descrição das teses e dissertações selecionadas na revisão narrativa. Santa Maria - Rio Grande do Sul, 2021.

\begin{tabular}{|c|c|c|}
\hline Cod & Autores & Título \\
\hline D1 & Melo (2018) & "Síndrome de burnout em enfermeiros assistenciais: Estudo em um hospital universitário". \\
\hline D2 & Nonino (2018) & $\begin{array}{l}\text { “. Indicadores da síndrome de burnout e presenteísmo em trabalhadores de enfermagem expostos } \\
\text { acidentalmente ao material biológico potencialmente contaminado". }\end{array}$ \\
\hline D3 & Oliveira (2018) & $\begin{array}{l}\text { "Relação da satisfação no trabalho, depressão e Síndrome de burnout na percepção da cultura de } \\
\text { segurança do paciente: estudo transversal com análise de modelagem por equações estruturais" }\end{array}$ \\
\hline D4 & Patricio (2018) & “Burnout, tensão emocional e depressão em profissionais de enfermagem em ambiente hospitalar". \\
\hline D5 & Soares (2018) & $\begin{array}{l}\text { "Burnout e fatores associados entre Profissionais de enfermagem de hospital municipal do Rio de } \\
\text { Janeiro". }\end{array}$ \\
\hline $\mathrm{T} 1$ & Almeida (2017) & $\begin{array}{l}\text { "Correlação entre clima organizacional, satisfação no trabalho e burnout em trabalhadores de } \\
\text { enfermagem". }\end{array}$ \\
\hline D6 & Freitas (2017) & $\begin{array}{l}\text { "A proposta de um plano de ação para redução do estresse em enfermeiros de um hospital } \\
\text { universitário". }\end{array}$ \\
\hline D7 & Lopes (2017) & $\begin{array}{l}\text { "Cultura de segurança do paciente e esgotamento em profissionais da saúde de um hospital } \\
\text { universitário de Manaus: Estudo transversal" }\end{array}$ \\
\hline $\mathrm{T} 2$ & Pereira (2017) & $\begin{array}{l}\text { "Variáveis mediadoras do burnout em profissionais de serviços de urgência e emergência: } \\
\text { aplicabilidade do Maslach Burnout Inventory - Human Services Survey (MBI-HSS)". }\end{array}$ \\
\hline D8 & Silva (2017) & "A síndrome de burnout: um estudo com profissionais de enfermagem de um hospital público". \\
\hline D9 & Vidotti (2017) & "Estresse. burnout e qualidade de vida na equipe de enfermagem". \\
\hline D10 & Bezerrro (2016) & "Estresse e síndrome de burnout nos enfermeiros de um hospital universitário". \\
\hline D11 & Silva (2016) & $\begin{array}{l}\text { "Verificar a existência de correlação entre o perfil cronobiológico e a ocorrência da síndrome de } \\
\text { burnout em enfermeiros assistenciais". }\end{array}$ \\
\hline $\mathrm{T} 3$ & Alves (2015) & "Ambiente de trabalho da enfermagem e segurança do paciente em unidades pediátricas". \\
\hline D12 & Candaten (2015) & $\begin{array}{l}\text { "Análise da associação entre a intenção de abandono da profissão e o estresse laboral em } \\
\text { enfermeiros de um hospital da serra gaúcha/RS". }\end{array}$ \\
\hline $\mathrm{T} 4$ & Sobral (2015) & "Fatores psicossociais de risco no trabalho e a síndrome de burnout". \\
\hline D13 & Fernandes (2015) & $\begin{array}{l}\text { "Associação entre síndrome de burnout, uso prejudicial de álcool e tabagismo em profissionais de } \\
\text { enfermagem nas unidades de terapia intensiva do Hospital das Clinicas de Botucatu". }\end{array}$ \\
\hline T5 & Gasparino (2015) & "Adaptação cultural e validação do practice environment scale para a cultura brasileira”. \\
\hline D14 & Gianini (2015) & $\begin{array}{l}\text { "Síndrome de burnout, ansiedade e depressão em profissionais da enfermagem de uma unidade de } \\
\text { urgência e emergência". }\end{array}$ \\
\hline D15 & Tondo (2015) & "Clima de segurança: percepção dos profissionais de enfermagem em um hospital de ensino". \\
\hline D16 & Sousa $(2015)$ & “. Síndrome de burnout em profissionais de enfermagem de um hospital público do Maranhão” \\
\hline D17 & Barrocas (2014) & $\begin{array}{l}\text { "Condições de trabalho de técnicos de enfermagem de um hospital particular: burnout e estratégias } \\
\text { defensivas". }\end{array}$ \\
\hline D18 & Oliveira (2014) & $\begin{array}{l}\text { "Síndrome de burnout em enfermeiros de um hospital público do município de Juazeiro do Norte - } \\
\text { CE'”. }\end{array}$ \\
\hline D19 & $\begin{array}{l}\text { Vasconcelos } \\
(2014)\end{array}$ & "Correlação do burnout e depressão em enfermeiros de unidade de terapia intensiva". \\
\hline D20 & Conto (2013) & $\begin{array}{l}\text { "Estresse laboral e suas implicações no processo de cuidar e do autocuidado da equipe de } \\
\text { enfermagem". }\end{array}$ \\
\hline D21 & Pereira (2013) & $\begin{array}{l}\text { “. Incidência da Síndrome de burnout em técnicos e auxiliares de enfermagem e sua associação } \\
\text { com o estresse precoce e estratégias de enfrentamento". }\end{array}$ \\
\hline D22 & Rieth (2013) & "Estresse e coping em trabalhadores de enfermagem de um hospital privado". \\
\hline D23 & Tito (2013) & $\begin{array}{l}\text { "Burnout e transtornos mentais comuns nos trabalhadores de enfermagem que assistem crianças } \\
\text { com cardiopatia grave". }\end{array}$ \\
\hline D24 & Neves (2012) & “. Impacto da satisfação no trabalho e da percepção de suporte organizacional sobre a Síndrome de \\
\hline
\end{tabular}




\begin{tabular}{|c|c|c|}
\hline & & burnout em trabalhadores de enfermagem de um Hospital Universitário". \\
\hline D25 & Dantas (2011) & $\begin{array}{l}\text { "Prevalência da Síndrome de burnout em enfermeiros da Rede Hospitalar de Urgência e } \\
\text { Emergência do Estado da Paraíba"" }\end{array}$ \\
\hline D26 & França (2011) & $\begin{array}{l}\text { "Estudo sobre síndrome de burnout em profissionais de enfermagem em hospitais de médio porte } \\
\text { no município de Cárceres-MT" }\end{array}$ \\
\hline D27 & Adriana (2011) & $\begin{array}{l}\text { "Síndrome de burnout: influências da satisfação no trabalho em profissionais de enfermagem de } \\
\text { um hospital público". }\end{array}$ \\
\hline D28 & Silva (2011) & $\begin{array}{l}\text { "O estresse laboral e a síndrome de burnout à luz dos sentimentos da enfermagem em centro } \\
\text { cirúrgico". }\end{array}$ \\
\hline D29 & Trigo (2011) & $\begin{array}{l}\text { "Validade fatorial do Maslach Burnout Inventory-Human Services Survey (MBI-HSS) em uma } \\
\text { amostra Brasileira de auxiliares de Enfermagem de um hospital universitário: influência da } \\
\text { depressão". }\end{array}$ \\
\hline D30 & Junior (2010) & "Burnout de enfermeiros: um estudo em hospitais de Belo Horizonte". \\
\hline D31 & Afecto (2008) & $\begin{array}{l}\text { "Avaliação do Estresse e da Síndrome de burnout em Enfermeiros que atuam em uma Unidade de } \\
\text { Terapia Intensiva". }\end{array}$ \\
\hline D32 & Marinho (2005) & "Estresse ocupacional e síndrome de burnout: um estudo em hospital privado". \\
\hline
\end{tabular}

D=Dissertação; T= Tese. Fonte: Elaboração própria.

\section{Discussão}

Assim, após a avaliação dos estudos relacionados no Quadro 1 emergiram três categorias temáticas: burnout e organização do trabalho; fatores associados ao burnout e estratégias e ações para prevenir o burnout; descritos na sequência.

Desta forma, em relação ao burnout e organização do trabalho, observou-se que um estudo com enfermeiros em dois hospitais de Belo Horizonte evidenciou que a carga horária semanal, entre 44 e 48 horas, favorece a despersonalização, a qual ocorre com maior frequência entre os homens. Mostrou, ainda, que enfermeiros com outro emprego apresentaram níveis maiores de exaustão emocional e que profissionais com mais de cinco anos de trabalho tinham escores mais elevados na realização pessoal. O gozo de férias apareceu como um fator de redução dos efeitos do burnout entre os participantes, principalmente na dimensão despersonalização (Chaves, 2010).

Um estudo com membros da equipe de enfermagem de um centro cirúrgico concluiu que os participantes gostam do que fazem em seu ambiente de trabalho e conseguem perceber quais são as principais fontes geradoras de estresse; aquilo que muitas vezes não é o trabalho em si, mas as situações envoltas ao setor, como a falta de materiais, equipamentos e insumos adequados para a prática de enfermagem (Silva, 2011).

Sobre a associação entre o burnout, turno de trabalho e estresse, observou-se que o estresse e as dimensões do burnout possuíam nível médio nos turnos diurno e noturno, sem prevalência de burnout (Bezerra, 2016).

Entretanto, uma pesquisa com enfermeiros, em relação ao burnout e serviço de emergência, expôs a presença do burnout no turno diurno, reflexo das condições e peculiaridades do trabalho de enfermagem nesse horário e tipo de serviço (Dantas, 2011).

Já outro estudo com profissionais da enfermagem apontou que a percepção do ambiente de trabalho pode influenciar de maneira positiva e negativa nos conceitos profissionais, na execução das atividades; fatos, que refletem diretamente na segurança do paciente (Tondo, 2015).

Nesta perspectiva, outros estudos com trabalhadores de enfermagem, sobre satisfação no trabalho, percepção do suporte organizacional e burnout, destacaram a importância do apoio organizacional e valorização dos trabalhadores, como ferramentas positivas para a segurança do paciente e redução do burnout (Tondo, 2015; Alves, 2015). 
Assim, uma pesquisa com 534 trabalhadores de enfermagem sobre a associação entre clima organizacional, satisfação no trabalho e burnout apresentou resultados estatisticamente significativos, haja vista que a baixa satisfação no trabalho ligado a um clima organizacional negativo são fatores de risco ao burnout (Almeida, 2017).

Neste sentido, observa-se que condições de trabalho favoráveis para a prática da enfermagem propiciam a redução do impacto na saúde dos profissionais da enfermagem, principalmente, no contato com o paciente em sofrimento e seus familiares (Barrocas, 2014).

Na mesma direção, um estudo com 52 enfermeiros, sobre metodologias de mudança do contexto organizacional que interferem no número de ocorrência do burnout, considerou relevante a reorganização do trabalho da enfermagem, pautado em ambientes físicos seguros e compatíveis com o trabalho desenvolvido, jornada de trabalho adequada, definição clara e transparente dos objetivos e metas institucionais; permitindo que este trabalhador tenha satisfação na prática da enfermagem (Sá, 2011).

Quanto aos fatores associados ao burnout, uma pesquisa com uma equipe de enfermagem verificou que o tradicional modelo de associação do burnout não é suficiente para explicar sua gênese; sugerindo medidas profiláticas para preservação da saúde (Sobral, 2015).

Nesta continuidade, estudos com enfermeiros comprovaram que quanto maior a sintomatologia depressiva, maior o nível de exaustão emocional, de despersonalização e menor a realização profissional; existe uma tendência de associação entre o desenvolvimento do burnout e a sintomatologia depressiva do trabalhador de enfermagem; (Vasconcelos, 2014; Pereira, 2015) destacando-se também a relevância do oferecimento de suporte emocional ao trabalhador da saúde (Marinho, 2005).

No entanto, outros estudos mostram relação entre o burnout, à ansiedade e a depressão em profissionais de enfermagem, associados a fatores organizacionais (Patrício, 2018; Gianini, 2015).

Com referência aos fatores associados ao burnout, um estudo com enfermeiros indicou a ocorrência dessa doença como um problema prevalente, multifatorial e carente de medidas preventivas. Neste contexto, constatou-se como fator de correlação significativa a atuação no horário de trabalho contrário ao perfil cronobiológico (Silva, 2016).

Por conseguinte, outras pesquisas com enfermeiros identificaram como fatores causadores de burnout, o estresse, a insuficiência de recursos materiais e humanos, limitações físicas, indefinição das atividades do enfermeiro e falta de educação continuada (Bezerra, 2016; Freitas, 2017).

Igualmente, estudos com enfermeiros demonstraram que esta população experiência situações estressantes no seu trabalho, vivenciando alguns sinais e sintomas, que podem levar o profissional a desenvolver o burnout (Tito, 2013; Afecto, 2008).

Com respeito à associação do burnout com o uso de drogas lícitas, observou-se entre os profissionais da área de Enfermagem, em unidades de terapia intensiva de um hospital universitário, a existência de associação do burnout com o consumo de álcool e tabaco. Outro estudo, denotou que a presença do burnout favorece a intenção de abandono da profissão e o estresse laboral em enfermeiros (Candaten, 2015).

Assim, estudos tem encontrado uma elevada prevalência de burnout entre trabalhadores da enfermagem, destacandose o desconhecimento da síndrome, a vulnerabilidade ao burnout e o alto nível de perda da realização pessoal (Pereira, 2015; Nonino, 2018; França, 2011).

Com referência as estratégias e ações para prevenir o burnout, destaca-se que a presença do burnout, entre os trabalhadores de enfermagem, instiga elaboração de estudos que sensibilizem a equipe de enfermagem, gestores e empregadores hospitalares a práticas laborais mais saudáveis (Bezerra, 2016; Oliveira, 2014). 
Nesse sentido, um estudo com uma equipe de enfermagem mostrou que investimentos para melhorar o ambiente de trabalho em unidades pediátricas podem reduzir os níveis de exaustão emocional, melhorar a satisfação do profissional de enfermagem e clima de segurança (Alves, 2015).

Nessa sequência, outra pesquisa, com profissionais de enfermagem, da área de infectologia e psiquiatria, indicou que a organização de um espaço para discussão sobre o trabalho é salutar e importante para a prática de enfermagem mais eficaz (Silva, 2017).

Igualmente, um estudo com 110 enfermeiros, que atuavam no setor de urgência e emergência, elucidou que o planejamento de estratégias que possibilitem à eliminação, redução e controle das variáveis associadas ao burnout favorecem o restabelecimento e a preservação da saúde mental nos trabalhadores (Oliveira, 2018).

Dessarte, um estudo, com enfermeiros, propôs ações de curto, médio e longo prazo, que causaram impacto positivo no cotidiano da enfermagem; entre as ações de curto prazo destaca-se o incentivo às reuniões semanais; de médio prazo a criação de locais para refeições e descanso; e de longo prazo, que a educação continuada na enfermagem deve ser empregada para atualização e capacitação dos profissionais (Freitas, 2017).

Nota-se que propostas de intervenção adequadas podem criar mudanças no ambiente de trabalho e consequentemente no profissional de enfermagem, gerando um ambiente de trabalho menos desgastante, mais produtivo e positivo (Tondo, 2015; Freitas, 2017; Benetti, 2013).

Isto posto, outro estudo, com profissionais da enfermagem, propôs duas direções para a implantação de medidas profiláticas a doenças laborais. A primeira, a direção organizacional, realizada com estratégias que contribuam para a minimização e redução de estresse, apoiando iniciativas sistemáticas de cuidado ao cuidador; a segunda a direção pessoal, construída através da adoção de medidas menos prejudiciais à saúde dos trabalhadores de enfermagem (Benetti, 2013; Conto, 2013).

Assim, reconhecer que as características que beneficiam e dificultam o desenvolvimento da práxis da enfermagem são definidas pelo ambiente de trabalho; e que identificar esta problemática proporcionará o alcance de melhores resultados, tanto para os pacientes como para os profissionais e instituições (Melo, 2018; Gasparino, 2015). Registra-se ainda um estudo que considerou o MBI como o principal instrumento de avaliação do burnout em trabalhadores da saúde (Pereira, 2017).

Os resultados encontrados nessa revisão contribuem com a pratica da enfermagem, pois mostraram os elementos associados ao burnout no ambiente hospitalar de trabalho da enfermagem, favorecendo a adoção de medidas que minimizem seus efeitos danosos à saúde.

\section{Conclusão}

Este estudo buscou identificar a tendência da produção científica brasileira sobre o burnout e enfermagem no ambiente hospitalar, nas dissertações e teses. Foi realizado o levantamento bibliográfico no Catálogo da CAPES, selecionandose 37 estudos para análise.

A tendência da produção científica sobre burnout entre trabalhadores de enfermagem focou em três aspectos principais, conforme categorias temáticas elencadas, isto é, à organização do trabalho e fatores associados, envolvendo aspectos de carga horária, tempo de trabalho, ter mais de um emprego, estresse, recursos materiais, dentre outros, e às estratégias e ações de prevenção do burnout. Dessa forma, observa-se que a tendência da produção científica sobre burnout tem sido relacionada á identificação de aspectos que explicam a ocorrência da síndrome na enfermagem, mas também tem avançado na elaboração e busca de implementar estratégias que possam evita-la entre os trabalhadores. 
Observou-se a ocorrência da associação entre o burnout e enfermagem no ambiente hospitalar; correlacionados, principalmente aos aspectos sociodemográficos laborais, a organização do trabalho da enfermagem, a baixa realização no trabalho e a exaustão profissional.

Os resultados encontrados evidenciam a necessidade de ações que favoreçam a prática da enfermagem em ambientes de trabalho mais éticos e positivos, o que contribuirá na melhoria da assistência de saúde e na redução de doenças laborais, como o burnout.

Neste sentido, a confecção de trabalhos científicos que enalteçam a eficácia destas ações, favorecerá tanto na pesquisa científica, quanto no ambiente de trabalho hospitalar, visto que, o enfoque da pesquisa na área da saúde do trabalhador baseiase no favorecimento de um local adequado, com clima ético positivo.

\section{Referências}

Abu Zied, M., Fekry, M., Mohsen, N., Morsey, M., El Serafy, D., \& Salah, M. (2020). Síndrome de burnout entre psiquiatras em hospital de saúde mental egípcio. Médio Oriente Atual Psiquiatria, 27(1), 25. https://doi.org/10.1186/s43045-020-00028-X

Afecto, M. D. C. P. (2008). Avaliação do Estresse e da Síndrome de Burnout em Enfermeiros que atuam em uma Unidade de Terapia Intensiva (Dissertação de Mestrado). Universidade de Guarulhos, Guarulhos, SP. http://tede.ung.br/bitstream/123456789/471/1/Maria+do+Carmo+Polonio+ Afecto_Disserta\%EF\% $\mathrm{BF} \% \mathrm{BD} \% \mathrm{EF} \% \mathrm{BF} \% \mathrm{BD} \% \mathrm{EF} \% \mathrm{BF} \% \mathrm{BD} \% \mathrm{EF} \% \mathrm{BF} \% \mathrm{BD}$.pdf

Almeida, M. C. D. S. (2017). Correlação entre clima organizacional, satisfação no trabalho e burnout em trabalhadores de enfermagem (Tese de Doutorado). Universidade de São Paulo, São Paulo, SP. https://teses.usp.br/teses/disponiveis/7/7140/tde-24092018-163120/en.php

Alvares, M. E. M., Thomas, E. B. A. F., Lamy, Z. C., Nina, R. V. A. H., Pereira, M. U. L., \& Garcia, J. B. S. (2020). Síndrome de Burnout entre Profissionais de Saúde nas Unidades de Terapia Intensiva: um estudo transversal com base populacional. Revista brasileira de terapia intensiva, 32(2), 251260. https://doi.org/10.5935/0103-507X.20200036

Alves, D. F. D. S. (2015). Ambiente de trabalho da enfermagem e segurança do paciente em unidades pediátricas (Dissertação de Mestrado). Universidade Estadual de Campinas, Campinas, SP. http://repositorio.unicamp.br/jspui/handle/REPOSIP/283864

Barrocas, P. H. N. (2014). Condições de trabalho de técnicos de enfermagem de um hospital particular: Burnout e estratégias defensivas (Dissertação de Mestrado). Universidade de Fortaleza, Fortaleza, CE. http://bdtd.ibict.br/vufind/Record/UFOR_eaca7fb5e29c4a0501ebeb831bd44b82

Benetti, E. R. R. (2013). Estresse e coping em trabalhadores de enfermagem de um hospital privado (Dissertação de Mestrado). Universidade Federal de Santa Maria, Santa Maria, RS. https://repositorio.ufsm.br/handle/1/7382

Bezerra, C. M. B. (2016). Estresse e síndrome de Burnout nos enfermeiros de um hospital universitário (Dissertação de Mestrado). Universidade Federal do Rio Grande do Norte, Natal, RN. https://repositorio.ufrn.br/handle/123456789/22002

Candaten, A. E. (2015). Análise da associação entre a intenção de abandono da profissão e o estresse laboral em enfermeiros de um hospital da serra gaúcha/RS (Dissertação de Mestrado). Universidade Federal Do Rio Grande Do Sul, Porto Alegre, RS. http://hdl.handle.net/10183/210209

Chaves, N. R., Jr. (2010). Burnout de enfermeiros: um estudo em hospitais de Belo Horizonte (Dissertação de Mestrado). Unihorizontes, Belo Horizonte, MG. https://mestrado.unihorizontes.br/burnout-de-enfermeiros-um-estudo-em-hospitais-de-belo-horizonte/

Conto, F. D. (2013). Estresse laboral e suas implicações no processo de cuidar e do autocuidado da equipe de enfermagem (Dissertação de Mestrado). Universidade Federal de Santa Catarina, Florianópolis, SC. https://repositorio.ufsc.br/handle/123456789/106828

Dantas, T. R. D. S. (2011). Prevalência da Síndrome de Burnout em enfermeiros da Rede Hospitalar de Urgência e Emergência do Estado da Paraíba (Dissertação de Mestrado). Universidade Federal da Paraíba, João Pessoa, PB. https://repositorio.ufpb.br/jspui/handle/tede/5121

Dorneles, A. J. A., Dalmolin, G. L., Andolhe, R., Magnago, T. S. B. S, \& Lunardi, V. L. (2020). Aspectos sociodemográficos e ocupacionais associados ao burnout em trabalhadores militares de enfermagem. Revista Brasileira de Enfermagem, 73(1) ed 20180350. https://doi.org/10.1590/0034-7167-2018-0350.

Dubale, B. W., Friedman, L. E., Chemali, Z., Denninger, J. W., Mehta, D. H., Alem, A., Fricchione, G. L., Dossett, M. L., \& Gelaye, B. (2019). Systematic review of burnout among healthcare providers in sub-Saharan Africa. BMC public health, 19(1),1247. https://doi.org/10.1186/s12889-019-7566-7.

Fernandes, L. S. (2015). Associação entre síndrome de burnout, uso prejudicial de álcool e tabagismo em profissionais de enfermagem nas unidades de terapia intensiva do Hospital das Clínicas de Botucatu - UNESP (Dissertação de Mestrado). Universidade Est.Paulista Júlio de Mesquita Filho/Botucatu, Botucatu, SP. https://repositorio.unesp.br/handle/11449/131898

França, F. M. D. (2011). Estudo sobre síndrome de burnout em profissionais de enfermagem em dois hospitais de médio porte no município de Cáceres - MT (Dissertação de Mestrado). Universidade de Brasília, Brasília, DF. http://repositorio.unb.br/handle/10482/8650

Freitas, M. D. A. A. (2017). Proposta de um plano de ação para redução do estresse em enfermeiros de um hospital universitário (Dissertação de Mestrado). Universidade Federal do Estado do Rio de Janeiro, Rio de Janeiro, RJ. http://docs.bvsalud.org/biblioref/2019/12/1026637/relatorio-de-pesquisa-dissertacaomariana-a-freitas-2017.pdf 
Gasparino, R. C. (2015). Adaptação cultural e validação do practice environment scale para a cultura brasileira (Dissertação de Mestrado). Universidade Estadual de Campinas, Campinas, SP. http://repositorio.unicamp.br/jspui/handle/REPOSIP/283903

Gianini, S. H. S. (2015). Síndrome de burnout, ansiedade e depressão em profissionais da enfermagem de uma unidade de urgência e emergência (Dissertação de Mestrado). Faculdade de Medicina de Marília, Marília, SP.https://sucupira.capes.gov.br/sucupira/public/consultas/coleta/ trabalhoConclusao/viewTrabalhoConclusao.jsf?popup=true\&id_trabalho=3364939

Gonçalves, R. L., \& Corrêa, L. M. (2020). Narrativas e tessituras adolescentes: metodologia e desafios de uma pesquisa(dora). Dialogia, 34,167-184. https://doi.org/10.5585/dialogia.n34.16706

Lopes, M. C. C. (2017). Cultura de segurança do paciente e esgotamento em profissionais da saúde de um hospital universitário de Manaus (Dissertação de Mestrado). Universidade Federal do Amazonas, Manaus, AM. https://tede.ufam.edu.br/handle/tede/6165

Marinho, R. D. C. (2005). Estresse ocupacional e síndrome de burnout: um estudo em hospital privado (Dissertação de Mestrado). Universidade De Taubaté, Taubaté, SP. https://www.yumpu.com/pt/document/view/34572886/estresse-ocupacional-estrategia-de-enfrentamento-e-ppgacombr

Martins, R., \& Tourinho, I. (2017). Pesquisa narrativa: interfaces entre histórias de vida, arte e educação. Santa Maria, RS: Fundação de Apoio a Tecnologia e Ciencia-Editora UFSM. https://books.google.com.br/books?id=u_gkDwAAQBAJ\&printsec=frontcover\&dq=Pesquisa+narrativa:+interfaces+entre+ hist $\%$ C3\%B3rias+de+vida,+arte+e+educa $\%$ C3\% A7\%C3\%A3o\&hl=ptBR\&ei=B83OYP_ZDqmcwQWP4fgCg\&cd=1\#v=onepage\&q=Pesquisa $\% 2$

Onarrativa \%3A\%20interfaces $\% 20$ entre $\% 20 \mathrm{hist} \% \mathrm{C} 3 \% \mathrm{~B} 3$ rias $\% 20 \mathrm{de} \% 20 \mathrm{vida} \% 2 \mathrm{C} \% 20 \mathrm{arte} \% 20 \mathrm{e} \% 20 \mathrm{educa} \% \mathrm{C} 3 \% \mathrm{~A} 7 \% \mathrm{C} 3 \% \mathrm{~A} 3 \mathrm{o} \& \mathrm{f}=\mathrm{false}$

Melo, F. M. D. A. B. (2018). Síndrome de burnout em enfermeiros assistenciais: estudo em um hospital universitário (Dissertação de Mestrado). Universidade Federal da Paraíba (João Pessoa), João Pessoa, PB. https://repositorio.ufpb.br/jspui/handle/123456789/15002

Neves, V. F. (2012). Impacto da satisfação no trabalho e da percepção de suporte organizacional sobre a Síndrome de Burnout em trabalhadores de enfermagem de um Hospital Universitário (Dissertação de Mestrado). Universidade Federal de Uberlândia, Uberlândia, MG. http://www.pgpsi.ip.ufu.br/node/296

Nonino, F. D. O. L. (2018). Indicadores da Síndrome de Burnout e presenteísmo em trabalhadores de enfermagem expostos acidentalmente ao material biológico potencialmente contaminado (Dissertação de Mestrado). Universidade De São Paulo, Ribeirão Preto, SP. https://teses.usp.br/teses/ disponiveis/22/22132/tde-29012019-101434/pt-br.php

Oliveira, A. M. D. (2018). Relação da satisfação no trabalho, depressão e Síndrome de Burnout na percepção da cultura de segurança do paciente: estudo transversal com análise de modelagem por equações estruturais (Dissertação de Mestrado). Universidade Estadual Paulista Júlio de Mesquita Filho, São Paulo, SP. http://hdl.handle.net/11449/153456

Oliveira, M. L. (2014). Síndrome de burnout em enfermeiros de um hospital público do município de Juazeiro do Norte - CE (Dissertação de Mestrado). Universidade Estadual de Feira de Santana, Feira de Santana, BA. https://drive.google.com/file/d/0B4_OV4G4QKDnRlAtVHlpXzhvZlFZaFQ5bn RIMGJNZE5nazdZ/ view?resourcekey=0-Z7BALcp3UFH3Y120k0WqGg

Patricio, D. F. (2018). Burnout, tensão emocional e depressão em profissionais de enfermagem em ambiente hospitalar (Dissertação de Mestrado). Universidade Estadual Da Paraíba, Campina Grande, PB. http://tede.bc.uepb.edu.br/jspui/handle/tede/3180

Pereira, S. D. S. (2013). Incidência da Síndrome de Burnout em técnicos e auxiliares de enfermagem e sua associação com o estresse precoce e estratégias de enfrentamento (Dissertação de Mestrado). Universidade de São Paulo/ Ribeirão Preto, Ribeirão Preto, SP. http://www.teses.usp.br/teses/disponiveis/ 22/22131/tde-25092013-153912/

Pereira, S. D. S. (2017). Variáveis mediadoras do Burnout em profissionais de serviços de urgência e emergência: aplicabilidade do Maslach Burnout Inventory - Human Services Survey (MBI-HSS) (Tese de Doutorado). Universidade de São Paulo (Ribeirão Preto), Ribeirão Preto, SP. https://www.teses.usp.br/teses/disponiveis/22/22131/tde-17082017-130158/pt-br.php

Porciuncula, A. M., Venâncio, A. S., \& Silva, C. M. F. P. (2020). Síndrome de Burnout em Gerentes da Estratégia de Saúde da Família. Ciência \& Saúde Coletiva, 25(4). https://doi.org/10.1590/1413-81232020254.22072018

Sá, A. M. (2011). Síndrome de burnout: influências da satisfação no trabalho em profissionais de enfermagem de um hospital público (Dissertação de Mestrado). Fundação Instituto Capixaba de Pesq. Em cont. Econ. E Finanças, Vitória, ES. http://legado.fucape.br/_public/producao_cientifica/8/ Dissertacao\%20Adriana\%20M\%C3\%BCller\%20Saleme\%20de\%20S\%C3\%A1.pdf

Silva, L. L. (2016). Existência de correlação entre o perfil cronobiológico e a ocorrência da síndrome de Burnout em enfermeiros assistenciais em um hospital público do município de Barueri - SP (Dissertação de Mestrado). Universidade Federal De São Paulo, São Paulo, SP. http://repositorio.unifesp.br/handle/11600/46487

Silva, R. J. D. (2017). A síndrome de burnout: um estudo com profissionais de enfermagem de um hospital público (Dissertação de Mestrado). Universidade Estadual de Mato Grosso do Sul, Dourados, MS. https://sucupira.capes.gov.br/sucupira/public/consultas/coleta/trabalhoConclusao/viewTrabalho Conclusao.jsf?popup $=$ true \&id_trabalho $=6088580$

Silva, V. L. (2011). O estresse laboral e a síndrome de Burnout à luz dos sentimentos da enfermagem em centro cirúrgico (Dissertação de Mestrado). Universidade Guarulhos, Guarulhos, SP. http://tede.ung.br/handle/123456789/201

Soares, R. D. S. (2018). Burnout e fatores associados entre profissionais de enfermagem de hospital municipal no Rio de Janeiro (Dissertação de Mestrado). Universidade Federal Fluminense, Niterói, RJ. https://app.uff.br/riuff/handle/1/7130

Sobral, R. C. (2015). Fatores psicossociais de risco no trabalho e a síndrome de Burnout (Tese de Doutorado). Universidade Estadual de Campinas, Campinas, SP. https://bdtd.ibict.br/vufind/Record/CAMP_4751feb6acaf489131d98f6b49b63fa4.

Sousa, A. R. B. (2015). Síndrome de Burnout em profissionais de enfermagem de um hospital público do Maranhão (Dissertação de Mestrado). Universidade Luterana Do Brasil, Canoas, RS. https://sucupira.capes.gov.br/sucupira/public/consultas/coleta/trabalho Conclusao/viewTrabalhoConclusao.jsf?popup $=$ true \&id_trabalho $=2448231$ 
Research, Society and Development, v. 10, n. 11, e548101120000, 2021

(CC BY 4.0) | ISSN 2525-3409 | DOI: http://dx.doi.org/10.33448/rsd-v10i11.20000

Tito, R. S. (2013). Burnout e transtornos mentais comuns nos trabalhadores de enfermagem que assistem crianças com cardiopatia grave (Dissertação de Mestrado). Universidade De São Paulo, São Paulo, SP. https://teses.usp.br/teses/disponiveis/7/7140/tde-14082013-132011/pt-br.php.

Tondo, J. C. A. (2015). Clima de segurança: percepção dos profissionais de enfermagem em um hospital de ensino (Dissertação de Mestrado). Universidade Estadual de Campinas, Campinas, SP. http://repositorio.unicamp.br/jspui/handle/REPOSIP/283902

Trigo, T. R. (2011). Validade fatorial do Maslach Burnout Inventory-Human Services Survey (MBI-HSS) em uma amostra Brasileira de auxiliares de Enfermagem de um hospital universitário: influência da depressão (Dissertação de Mestrado). Universidade de São Paulo, São Paulo, SP. https://www.teses.usp.br/teses/disponiveis/5/5142/tde-26052011-123120/pt-br.php

Vasconcelos, E. M. D. (2014). Correlação do burnout e depressão em enfermeiros de unidade de terapia intensiva (Dissertação de Mestrado). Universidade Federal de São Paulo, São Paulo, SP. https://repositorio.unifesp.br/handle/11600/47399

Vidotti, V. (2017). Estresse, burnout e qualidade de vida na equipe de enfermagem (Dissertação de Mestrado). Universidade Estadual de Londrina, Londrina, PR. http://www.bibliotecadigital.uel.br/document/?code=vtls000216208

Winskell, K., Singleton, R., \& Sabben, G. (2018). Enabling Analysis of Big, Thick, Long, and Wide Data: Data Management for the Analysis of a Large Longitudinal and Cross-National Narrative. Qualitative health research, 28(10),1629-1639. https://doi.org/10.1177/1049732318759658 